\title{
MORFO-ANATOMÍA DE PLÁNTULAS EN ESPECIES DE PACHYCEREEAE: ¿HASTA CUÁNDO SON PLÁNTULAS?
}

\author{
Sofía Loza-Cornejo ${ }^{1,3}$ y Teresa Terrazas ${ }^{2}$ \\ 1Programa de Botánica, Colegio de Postgraduados, dirección actual: Centro Universitario de Los Lagos, \\ Universidad de Guadalajara \\ 22Departamento de Botánica, Instituto de Biología, Universidad Nacional Autónoma de México \\ ${ }^{3}$ Autor para la correspondencia: sofialo@culagos.udg.mx, sloza49@hotmail.com
}

\begin{abstract}
Resumen: Se describe y compara la morfo-anatomía de plántulas de la tribu Pachycereeae con el objetivo de establecer diferencias entre las fases del crecimiento inicial y establecimiento y distinguir así la diferenciación entre plántula e individuo juvenil. El registro de los cambios morfo-anatómicos se obtuvo de una muestra de más de 500 plántulas de seis especies representantes de cinco géneros de esta tribu, de las cuales se tomaron semanalmente tres plántulas durante los tres primeros meses y posteriormente cada mes hasta completar 24 meses de crecimiento, con el propósito de registrar sus cambios morfo-anatómicos. Se encontraron diferencias $(P<0.05)$ para altura de la plántula, longitud de la raíz, altura y diámetro del tallo entre las especies en las diferentes edades. El retardo en el desarrollo del tallo y la raíz fue característico de Escontria chiotilla y Myrtillocactus geometrizans provenientes de semillas pequeñas. Las especies comparten atributos de los tejidos dérmico, fundamental y vascular, pero se destacan los cuerpos de proteína en Pachycereus grandis y los cristales prismáticos en epidermis-hipodermis de las especies de Neobuxbaumia. Al establecerse el cámbium vascular sólo se diferencian elementos de vaso y traqueidas de banda ancha, y las fibras aparecen hasta después del año de edad en algunas especies. Se concluye que la desaparición de cotiledones, presencia de costillas, establecimiento de cámbium vascular, mayor acumulación de xilema secundario y diferenciación de haces vasculares medulares permiten establecer el límite entre la etapa de plántula y planta joven en Pachycereeae.

Palabras clave: cristales, epicótilo, Escontria chiotilla, hipocótilo, Myrtillocactus geometrizans, Neobuxbaumia mezcalaensis, Neobuxbaumia multiareolata, Pachycereeae, Pachycereus grandis, plántulas, Stenocereus queretaroensis, traqueidas de banda ancha.
\end{abstract}

\begin{abstract}
Morpho-anatomy of species in Tribe Pachycereeae seedlings is described and compared with the aim to find differences between the initial growth and establishment stages and to determine the point in time of the change from seedling to young plant. The morpho-anatomical traits were observed from sampling more than 500 seedlings of six species belonging to five genera of this tribe. Seedlings were collected weekly the first three-months after germination and afterwards they were collected monthly to complete 24-months of growth. Statistical differences $(P<0.05)$ were observed for seedling height, root length, and stem width and height between the species at different ages. A delay in stem and root development was found in Escontria chiotilla and Myrtillocactus geometrizans, species with the smallest seeds. Moreover, the studied species share dermal, fundamental, and vascular tissue traits, however the protein bodies distinguish Pachycereus grandis and the prismatic crystals in epidermis-hypodermis characterize species of Neobuxbaumia. At the onset of vascular cambium, only vessel elements and wide band tracheids become differentiated, whereas fibers appeared in some species after they had one-year of age. We concluded that disappearance of cotyledons, differentiation of ribs, establishment of vascular cambium, a greater accumulation wood, and differentiation of medullary bundles allow us to determine the limits between the seedling stage and the young plant stage.

Key words: crystals, epicotyl, Escontria chiotilla, hypocotyl, Myrtillocactus geometrizans, Neobuxbaumia mezcalaensis, Neobuxbaumia multiareolata, Pachycereeae, Pachycereus grandis, Stenocereus queretaroensis, seedlings, wide-band-tracheids.
\end{abstract}

a ontogenia también llamada morfogénesis se define - como el desarrollo de un organismo o de uno de sus componentes (órganos, tejidos, células) y su estudio constituye una fuente de evidencia para la interpretación morfológica de las especies (Pérez-García y Mendoza, 2002). La ca- racterización morfológica de plántulas de diversas especies ofrece la posibilidad de conocer la existencia de estructuras u órganos de etapas juveniles, que desaparecen al continuar el desarrollo y que pueden tener valor para establecer relaciones de parentesco (Burger, 1972; De Vogel, 1980; Ricar- 
di et al., 1987; Zevallos y Flores, 2003; San-Miguel-Chávez et al., 2006; Leroy et al., 2010).

En Cactaceae se reconocen actualmente cuatro subfamilias, siendo Cactoideae la subfamilia más grande (Anderson, 2001). La tribu Pachycereeae pertenece a Cactoideae, de acuerdo a Anderson tiene 17 géneros y cerca de 166 especies (Anderson, 2001). Las características morfo-anatómicas de plantas adultas de Cactaceae como la forma y orientación de los tallos y la presencia de hojas modificadas en espinas, se han interpretado como adaptaciones a los ambientes áridos o semiáridos (Gibson y Nobel, 1986; Mauseth, 2006). Las modificaciones anatómicas más notorias son la presencia de ceras epicuticulares, cutícula gruesa, epidermis múltiple, hipodermis colenquimatosa y desarrollo de grandes proporciones de tejido medular y cortical con células de mucílago (Terrazas y Mauseth, 2002; Mauseth, 2006), así como la presencia de las traqueidas de banda ancha en el xilema secundario (Mauseth, 2006). Sin embargo, cuáles de estas características se expresan desde el estado de plántula es desconocido para Pachycereeae. En qué momento de la ontogenia se presentan estas características adaptativas en las cactáceas es poco conocido. Los estudios sobre la ontogenia de sus plántulas se han centrado en describir aspectos de su morfología (Ganong, 1898; Meyrán, 1956; Hamilton, 1970b; Gibson, 1988a, b, 1989a, b, 1990a, b; Gutiérrez de la Rosa et al., 1999). Buxbaum (1961), en su monografía de la tribu Pachycereeae, enfatizó la necesidad de estudiar comparativamente las plántulas ya que sus diferencias pueden tener valor taxonómico. Otros estudios en plántulas se han centrado en conocer los mecanismos morfogenéticos que controlan el desarrollo de su meristemo apical (Mauseth, 1978, 1979, 1980); de cloroplastos en hojas de especies de Opuntia (Freeman, 1973); de tubérculos y espinas de $\mathrm{Pe}$ lecyphora y Encephalocarpus (Boke, 1959), del desarrollo de la raíz primaria y secundarias (Dubrovsky, 1997; Dubrovsky et al., 1998), de la relación morfo-anatómica con aspectos de su metabolismo fotosintético (Loza-Cornejo et al., 2003) y de la presencia de traqueidas de banda ancha (Loza-Cornejo et al., 2003; Mauseth, 2004; RomeroGodofredo y Melo-de Pinna, 2008). ¿Existe homogeneidad morfo-anatómica en las plántulas de Pachycereeae?, ¿los caracteres morfo-anatómicos de estas plántulas representan alguna adaptación importante para su establecimiento y crecimiento inicial como se ha postulado para la fase adulta? y ¿qué atributos morfo-anatómicos permiten reconocer el fin del estadio de plántula en las especies de esta tribu? En el presente trabajo se describen y comparan los caracteres morfo-anatómicos de plántulas de seis especies de Pachycereeae con la finalidad de establecer diferencias entre las fases de crecimiento inicial y establecimiento, identificar que adaptaciones se adquieren desde esta fase del ciclo de vida y establecer hasta que momento se presenta el estadio de plántula en las especies estudiadas.

\section{Materiales y Métodos}

Se recolectaron durante los meses de abril y julio de 2000, frutos maduros de tres individuos de seis especies representativas de la tribu Pachycereeae en sus áreas de distribución (Escontria chiotilla (F.A.C. Weber ex K.Schum.) Rose, Guerrero, Xalitlán, col. Terrazas 407; Myrtillocactus geometrizans (Mart. ex Pfeiff.) Console, Querétaro, Higuerillas, Terrazas 555; Neobuxbaumia mezcalaensis (Bravo) Backeb., Guerrero, Cañón del Zopilote, Terrazas 404; Neobuxbaumia multiareolata (E.Y. Dawson) Bravo, Scheinvar \& SánchezMej., Guerrero, Tierra Colorada, Terrazas 537; Pachycereus grandis Rose, Puebla, Acatlán, Arias 1380; Stenocereus queretaroensis (F.A.C. Weber) Buxb., Jalisco, Sayula, Arreola-Nava 1342. Los ejemplares de respaldo están depositados en el herbario del Colegio de Postgraduados, CHAPA. Los frutos fueron disectados para obtener las semillas, las cuales se desinfectaron por inmersión en hipoclorito de sodio comercial al 10\% durante $5 \mathrm{~min}$ (Vega-Villasante et al., 1996) y se sembraron sobre papel filtro saturado con agua destilada en cajas de petri estériles (Dubrovsky, 1996). Ninguna de las especies tuvo problemas en su respuesta germinativa (Loza-Cornejo et al., 2008).

Las plántulas obtenidas se transplantaron a charolas con una mezcla de agrolita-tezontle-tierra para maceta, 1:1:1 y se mantuvieron en un invernadero donde continuó su crecimiento con riego dos veces por semana. Durante los primeros tres meses después de la germinación, las plántulas fueron recolectadas cada semana y posteriormente cada mes hasta completar un periodo de crecimiento de 24 meses. Las plántulas recolectadas (tres repeticiones por fecha, cada semana/tres meses y cada mes/24 meses) se fijaron en

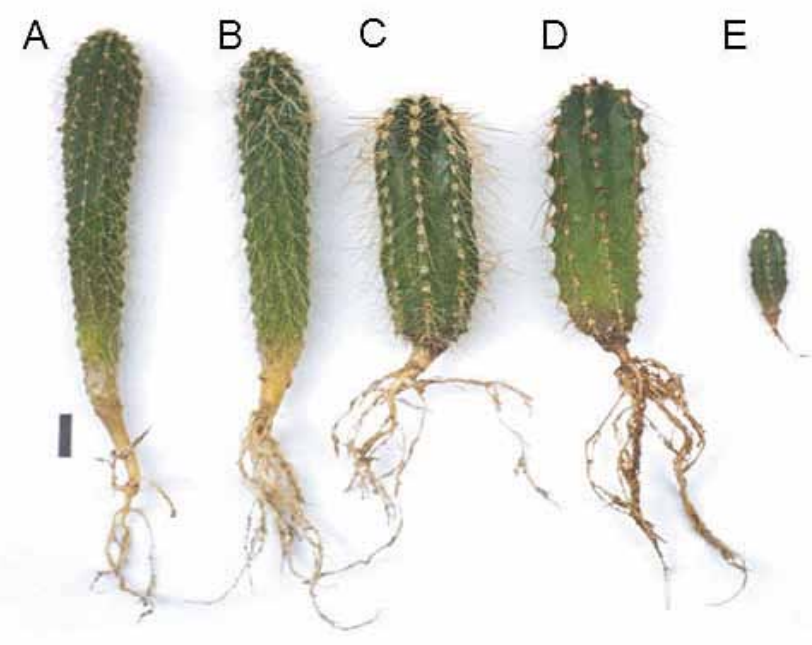

Figura 1. Plantas jóvenes de 24 meses de edad de algunas especies de Pachycereeae. A. Neobuxbaumia multiareolata; B. N. mezcalaensis; C. Pachycereus grandis; D. Stenocereus queretaroensis; E. Myrtillocactus geometrizans. Escala $=1 \mathrm{~cm}$. 
solución de Navashin (Berlyn y Miksche, 1976). La medición de caracteres morfológicos de los diferentes órganos de las plántulas se realizó con un vernier digital Mitutoyo Digimatic SR44. Posterior a la descripción morfológica, las mismas plántulas se fraccionaron y deshidrataron en un cambiador automático Leica TP1020 con alcohol terbutílico (TBA, 10\%-100\%) por cuatro horas en cada concentración, finalizando con dos cambios de parafina Leica $\left(56-58^{\circ} \mathrm{C}\right)$. Una vez incluidas las muestras en parafina se hicieron cortes transversales y longitudinales en un micrótomo rotatorio a 12-14 $\mu \mathrm{m}$ de grosor. La descripción de caracteres anatómicos de los tejidos dérmico, fundamental y vascular, se realizó utilizando un microscopio Olympus BX50. Los datos cuantitativos fueron sintetizados mediante la estadística de la tendencia central y de dispersión para cada muestreo, y se buscaron diferencias para los caracteres morfológicos entre edades y entre especies mediante el análisis de varianza del modelo general lineal seguido de una comparación múltiple de medias de Tukey (SAS Institute, 2008).

\section{Resultados}

Morfología de plántulas. Con la germinación surge la radícula apenas distinguible del hipocótilo porque ambos tienen coloración blanquecina o verde pálido. Los cotiledones son pequeños angostos, color verde, forma triangular y ligeramente separados entre sí; con el ápice de forma variable, redondos en las especies de Neobuxbaumia hasta puntiagudos en Myrtillocactus geometrizans. Pachycereus grandis presenta cotiledones desiguales, más suculentos y de mayor tamaño que el resto de las especies estudiadas. Después de dos semanas de crecimiento, el eje hipocótiloraíz es una estructura corta, de forma ovoide o más o menos cónica y presenta en su base la raíz primaria, corta, delgada, sin ramificaciones y de color amarillo pálido o blanquecino con numerosos pelos radicales. A esa edad las plántulas de Neobuxbaumia mezcalaensis y Stenocereus queretaroensis se diferencian del resto de las especies por presentar coloración rojiza en el eje hipocótilo-raíz. El epicótilo se observa sólo microscópicamente como una pequeña protuberancia en especies de Neobuxbaumia y S. queretaroensis a las dos semanas de edad; en plántulas de un mes de edad de $P$. grandis y a los dos meses en Escontria chiotilla; mientras que en M. geometrizans se evidencia a los cuatro meses de edad.

A los seis meses, en el epicótilo se inicia la formación de podarios; dos alternan con los cotiledones al inicio y con la nueva formación de tubérculos, éstos siguen una disposición dispersa. Los tubérculos son pequeños, aplanados y con sus aréolas terminales. Las primeras aréolas tienen espinas diminutas y tricomas blanquecinos. Entre el primer y doceavo mes de crecimiento, el epicótilo inicialmente formado como un abultamiento corto y globoso, se distingue ya como una estructura cilíndrica con hileras desordenadas de tubérculos. Entre los 12 y 14 ó 24 meses de edad, las plántulas adquieren la forma de cilindro corto en el cual se inicia la formación de las primeras costillas por alineación vertical de tubérculos (Figura 1). A los 24 meses de edad el epicótilo (tallo) representa más del 50\% del cuerpo total de la plántula y las costillas varían en número, de cinco en Escontria chiotilla y Myrtillocactus geometrizans, ocho en Pachycereus grandis y Stenocereus queretaroensis hasta 12 ó 13 en Neobuxbaumia mezcalaensis y N. multiareolata. La reducción de los cotiledones se inicia a los 12 meses en $M$. geometrizans y $S$. queretaroensis y hasta los 24 meses en $E$. chiotilla. La longitud de la raíz se incrementa con la edad de la plántula, diferenciándose la raíz primaria y varias raíces laterales de la misma longitud (Figura 1) hasta los 24 meses de edad.

Comparación interespecífica de caracteres morfológicos. Se encontraron diferencias para altura total de plántulas $(F=$ 31.38-204.76, $g l=5, P<0$. 0001, $N=18$ ) y longitud de la raíz $(F=12.42-24.36, g l=5, P<0.0001, N=18)$ entre especies para las diferentes edades (Cuadros 1,2). El desarrollo del epicótilo alcanza menos de $2 \mathrm{~cm}$ a los cuatro meses de edad en cuatro de las seis especies estudiadas (Figura 2).
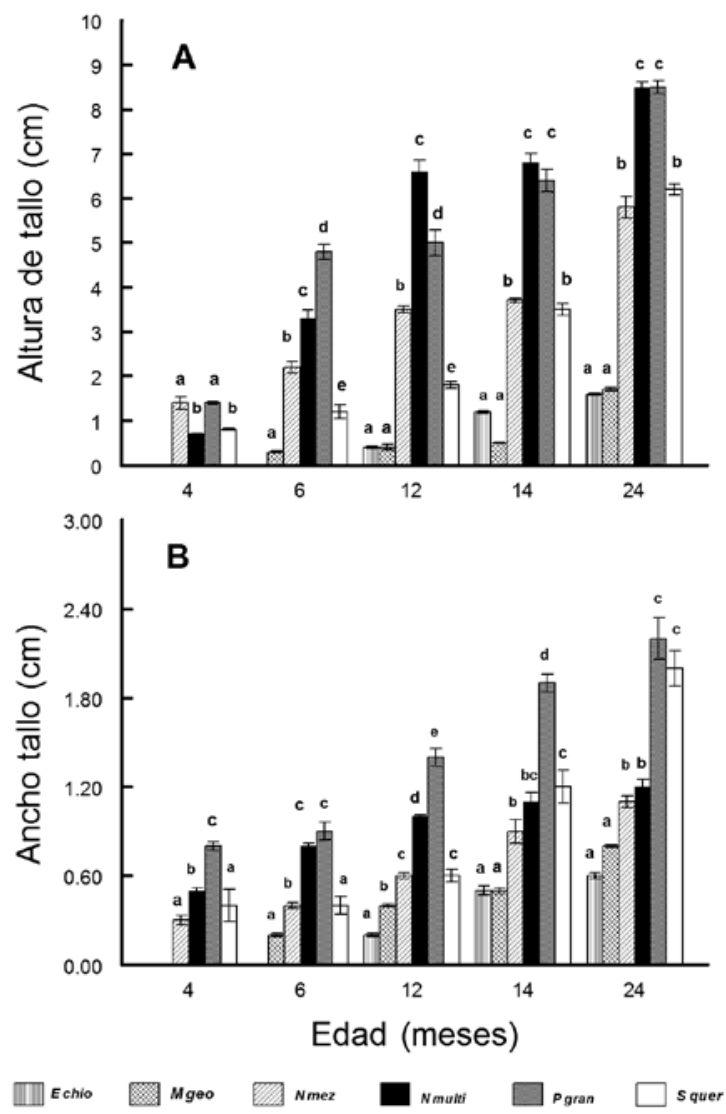

Figura 2. Valores de la media del tamaño del tallo de plántulas de Pachycereeae a diferentes edades. A. Altura $(\mathrm{cm}) ;$ B. Ancho $(\mathrm{cm})$. Las barras representan la media de tres repeticiones \pm 1 error estándar. Letras diferentes indican diferencias estadísticamente significativas entre las especies $(P<0.05$, Tukey). 
Cuadro 1. Media \pm error estándar de la altura total de plántulas $(\mathrm{en} \mathrm{cm}$ ) a diferentes edades en seis especies de Pachycereeae.

\begin{tabular}{|c|c|c|c|c|}
\hline \multirow[t]{2}{*}{ Especie } & \multicolumn{4}{|c|}{ Edad (meses) } \\
\hline & 0.5 & 6 & 12 & 24 \\
\hline Escontria chiotilla & $0.6 \pm 0.03^{a}$ & $0.8 \pm 0.02^{a}$ & $1.6 \pm 0.08^{a}$ & $2.1 \pm 0.08^{a}$ \\
\hline Myrtillocactus geometrizans & $0.5 \pm 0.02^{a}$ & $0.8 \pm 0.02^{a}$ & $1.2 \pm 0.08^{a}$ & $3.3 \pm 0.09^{a}$ \\
\hline Neobuxbaumia mezcalaensis & $0.9 \pm 0.33^{a}$ & $2.6 \pm 0.14^{b}$ & $4.5 \pm 0.5^{b}$ & $9.8 \pm 0.8^{b}$ \\
\hline N. multiareolata & $1.7 \pm 0.36^{b}$ & $6.9 \pm 0.16^{c}$ & $11.7 \pm 0.9^{\mathrm{c}}$ & $15.5 \pm 0.23^{c}$ \\
\hline Pachycereus grandis & $2.9 \pm 0.16^{\mathrm{c}}$ & $7.7 \pm 0.44^{\mathrm{c}}$ & $9.7 \pm 0.61^{\mathrm{c}}$ & $15.7 \pm 0.88^{c}$ \\
\hline Stenocereus queretaroensis & $1.1 \pm 0.27 \mathrm{ab}$ & $2.1 \pm 0.18^{b}$ & $4.6 \pm 0.16^{b}$ & $12.0 \pm 0.6^{b}$ \\
\hline
\end{tabular}

Medias con letras diferentes indican diferencias estadísticamente significativas entre las especies $(P<0.05$, Tukey).

Cuadro 2. Media \pm error estándar de longitud de la raíz (en cm) de plántulas a diferentes edades en seis especies de Pachycereeae.

\begin{tabular}{lcccc}
\hline \multicolumn{1}{c}{ Especie } & \multicolumn{2}{c}{ Edad (meses) } \\
\hline & 0.5 & 6 & 12 & 24 \\
Escontria chiotilla & $0.25 \pm 0.0^{\mathrm{ab}}$ & $0.43 \pm 0.02^{\mathrm{a}}$ & $0.69 \pm 0.01^{\mathrm{a}}$ & $0.84 \pm 0.03^{\mathrm{a}}$ \\
Myrtillocactus geometrizans & $0.21 \pm 0.03^{\mathrm{ab}}$ & $0.28 \pm 0.08^{\mathrm{a}}$ & $0.42^{\mathrm{a}} \pm .05^{\mathrm{a}}$ & $1.20 \pm 0.03^{\mathrm{ab}}$ \\
Neobuxbaumia mezcalaensis & $0.11 \pm 0.07^{\mathrm{a}}$ & $1.55 \pm 0.08^{\mathrm{b}}$ & $3.20 \pm 0.1^{\mathrm{b}}$ & $3.80 \pm 0.37^{\mathrm{bc}}$ \\
N. multiareolata & $0.65 \pm 0.2^{\mathrm{bc}}$ & $1.52 \pm 0.30^{\mathrm{b}}$ & $4.60 \pm 0.32^{\mathrm{b}}$ & $5.20 \pm 0.21^{\mathrm{c}}$ \\
Pachycereus grandis & $1.10 \pm 0.11^{\mathrm{c}}$ & $3.80 \pm 0.17^{\mathrm{c}}$ & $4.50 \pm 0.69^{\mathrm{b}}$ & $8.50 \pm 1.43^{\mathrm{d}}$ \\
Stenocereus queretaroensis & $0.25 \pm 0.1^{\mathrm{b}}$ & $0.75 \pm 0.12^{\mathrm{a}}$ & $2.90 \pm 0.21^{\mathrm{b}}$ & $6.40 \pm 0.23^{\mathrm{cd}}$ \\
\hline
\end{tabular}

Medias con letras diferentes indican diferencias estadísticamente significativas entre las especies $(P<0.05$, Tukey).

En Escontria chiotilla y Myrtillocactus geometrizans no se reconoce el epicótilo hasta los 6 meses o más de edad. La comparación interespecífica para altura $(F=89.34-204.76$, $g l=5, P<0.0001, N=18)$ y para diámetro $(F=53.86$ 228.29, $g l=5, P<0.0001, N=18$ ) del epicótilo-tallo en plántulas de diferentes edades mostró diferencias entre algunas especies (Figura 2).

Anatomía del embrión y meristemo apical de la plántula. Las plántulas de 1 a 2 semanas de edad muestran un meristemo apical más o menos plano, diferenciado por una túnica de un único estrato celular, con núcleos prominentes y un corpus con 3 a 4 estratos de células (Figura 3A). Las diferentes zonas del meristemo apical van desarrollándose de manera gradual y en las plántulas de un mes de edad ya se distinguen las siguientes regiones: una túnica claramente definida y el corpus con zona de células madre centrales, zona periférica y zona meristemática médula-costilla. Se observan cuerpos de proteína amorfos en Pachycereus grandis (Figura 3B) y granos de almidón en el tejido fundamental en especies de Neobuxbaumia. Es notoria la diferenciación de elementos vasculares a partir del procámbium, el tejido vascular procambial forma cadenas cortas a lo largo del eje longitudinal hipocótilo-raíz y en la base de los cotiledones. Raíz.-. Al inicio de la germinación, la radícula se distingue por presentar una cofia pequeña en su extremo apical. Al- gunas células de la epidermis forman pelos radicales cortos persistentes durante 7 a 10 días después de la germinación (Figura 3C, D). Subyacente a la epidermis hay parénquima, endodermis y 1 ó 2 hileras de células en el periciclo. El xilema tiene desarrollo exarco y presenta escasos elementos traqueales estrechos de paredes secundarias con engrosamientos anulares y helicoidales (Figura 3E) y placa de perforación simple. Los primordios de raíces laterales se originan tempranamente a partir de células del periciclo de la raíz primaria y a distancias variables del meristemo radical. En poco tiempo la raíz tiene crecimiento secundario, formando simultáneamente cámbium vascular y felógeno. Las primeras divisiones periclinales entre xilema y floema y posteriormente en parénquima exterior al protoxilema, constituyen una evidencia del inicio de actividad cambial. El xilema secundario presenta elementos de vaso de paredes secundarias con engrosamientos helicoidal-reticulados y placa de perforación simple, parénquima axial y fibras libriformes nucleadas. En el floema secundario hay elementos de tubo criboso y células acompañantes. La cantidad de tejido vascular secundario es mayor en la raíz y a diferencia del hipocótilo se forma un cilindro sólido de xilema (Figura $3 \mathrm{~F}$ ). La peridermis se origina de células suprayacentes al periciclo, a partir de donde el felógeno diferencia varios estratos de felema que se colapsan desde antes de un mes de 


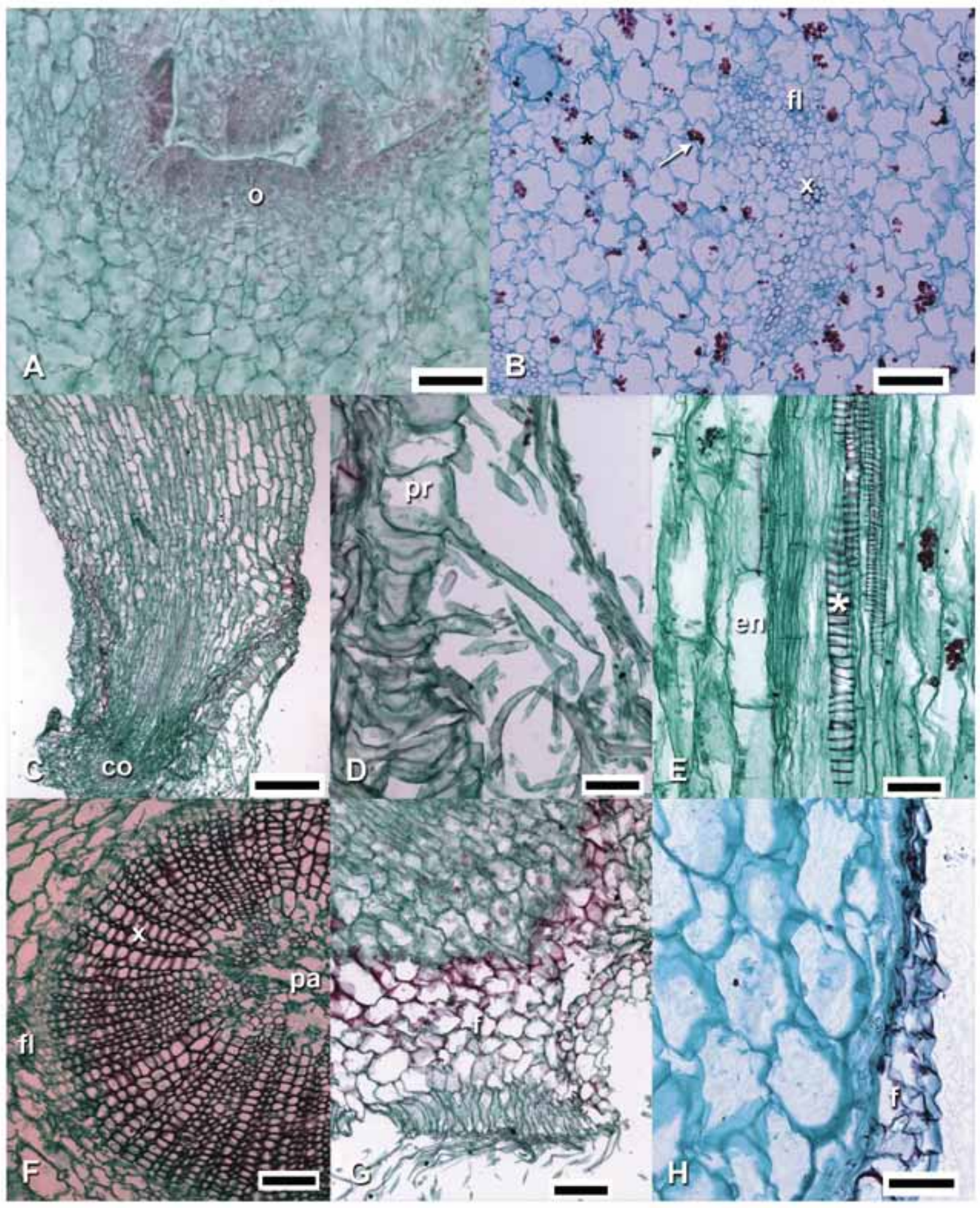

Figura 3. Meristemo apical y radícula-raíz en plántulas de Pachycereeae, cortes longitudinales (L) y transversales (T). A. Meristemo apical (L) en Pachycereus grandis, cinco días de edad. B. Hipocótilo (T) tejido vascular y cuerpos de proteína (flecha) en Pachycereus grandis, diez días de edad. C. Radícula (L) en Neobuxbaumia mezcalaensis, siete días de edad. D. Pelos radicales (L) en Neobuxbaumia multiareolata, siete días de edad. E. Tejido vascular y endodermis (L) en Pachycereus grandis, un mes de edad. F. Raíz (T) con crecimiento secundario Neobuxbaumia mezcalaensis, 14 meses de edad. G. Felema en raíz (L) Neobuxbaumia multiareolata, diez días de edad. H. Felema en raíz (T) en Escontria chiotilla, un mes de edad. Barra de $50 \mu \mathrm{m}$ en A, D, E, H; de $100 \mu \mathrm{m}$ en B, F, G; de $300 \mu \mathrm{m}$ en C. co, cofia; en, endodermis; f, felema; fl, floema; o, corpus; pa, parénquima; pr, pelo radical; x, xilema; *, elementos traqueales. 


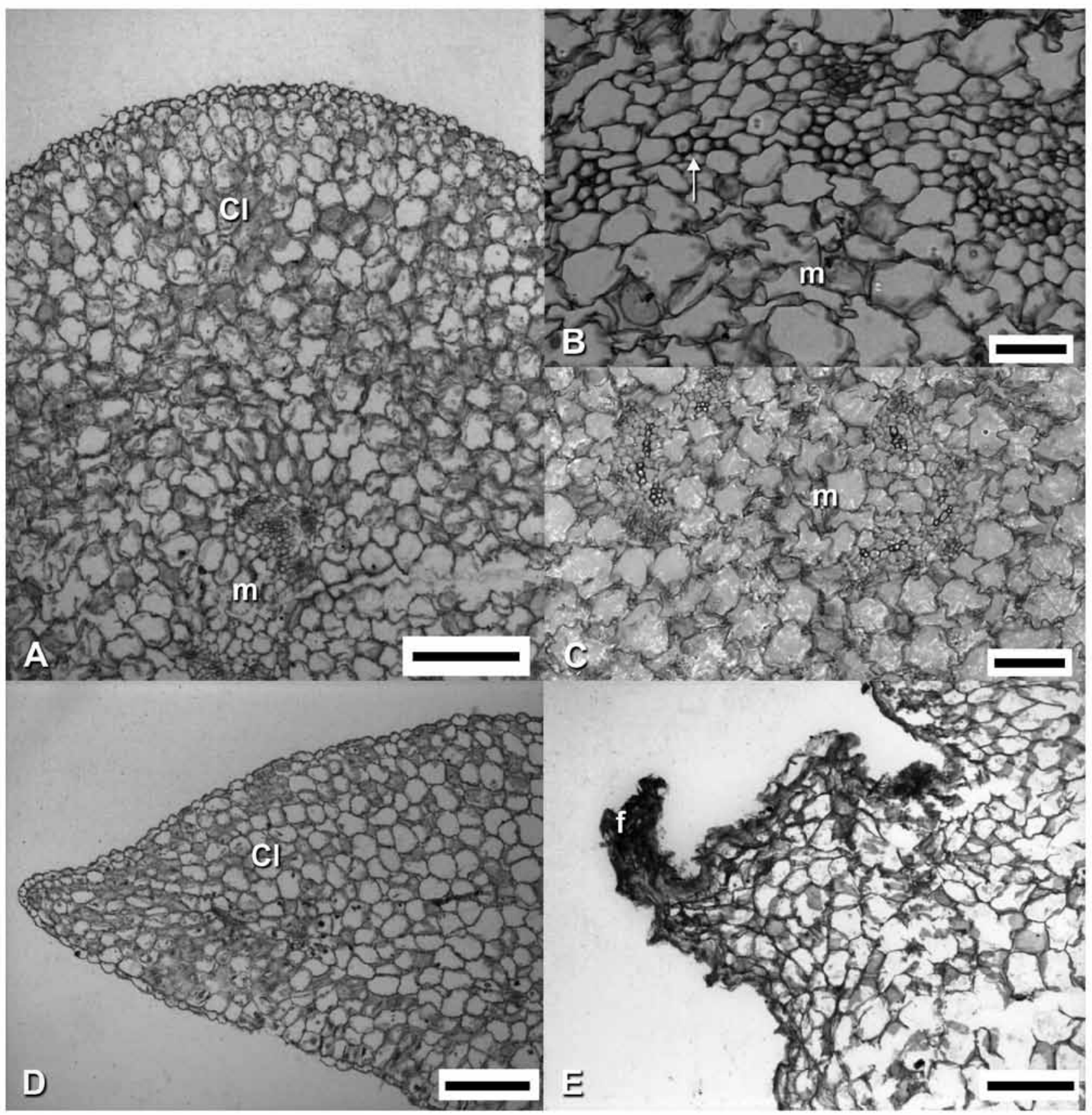

Figura 4. Hipocótilo y cotiledones en plántulas de Pachycereeae, cortes transversales. A. Médula y córtex en Neobuxbaumia multiareolata, diez días de edad. B. Transición del tejido vascular (flecha = xilema) en Pachycereus grandis, un mes de edad. C. Cilindro vascular con ocho haces en Neobuxbaumia mezcalaensis, un mes de edad. D. Cotiledón en Neobuxbaumia multiareolata, diez días de edad. E. Cotiledón con peridermis, Neobuxbaumia mezcalaensis, 14 meses de edad. Barra de $300 \mu \mathrm{m}$ en A, D, E; de $50 \mu \mathrm{m}$ en B; de $100 \mu \mathrm{m}$ en C. $\mathrm{Cl}$, clorénquima; f, felema; $\mathrm{m}$, médula.

edad de las plántulas (Figura 3G, H).

Eje hipocótilo-raíz.- La epidermis es simple con paredes anticlinales onduladas, vista en superficie, y algunos estomas. La médula y tejido cortical presentan células de parénquima sin contenidos minerales (Figura 4A). En el protoxilema de plántulas desde un mes de edad, se distinguen elementos traqueales ( 3 a 4 vasos) distribuidos en dos hileras de tejido vascular separadas entre sí por parénquima que presenta granos de almidón únicamente en Pachycereus grandis. El desarrollo del xilema en el hipocótilo cambia de exarco a endarco, formando dos haces vasculares colaterales en plántulas de mayor edad (Figura 4B, C). El lugar de origen del 


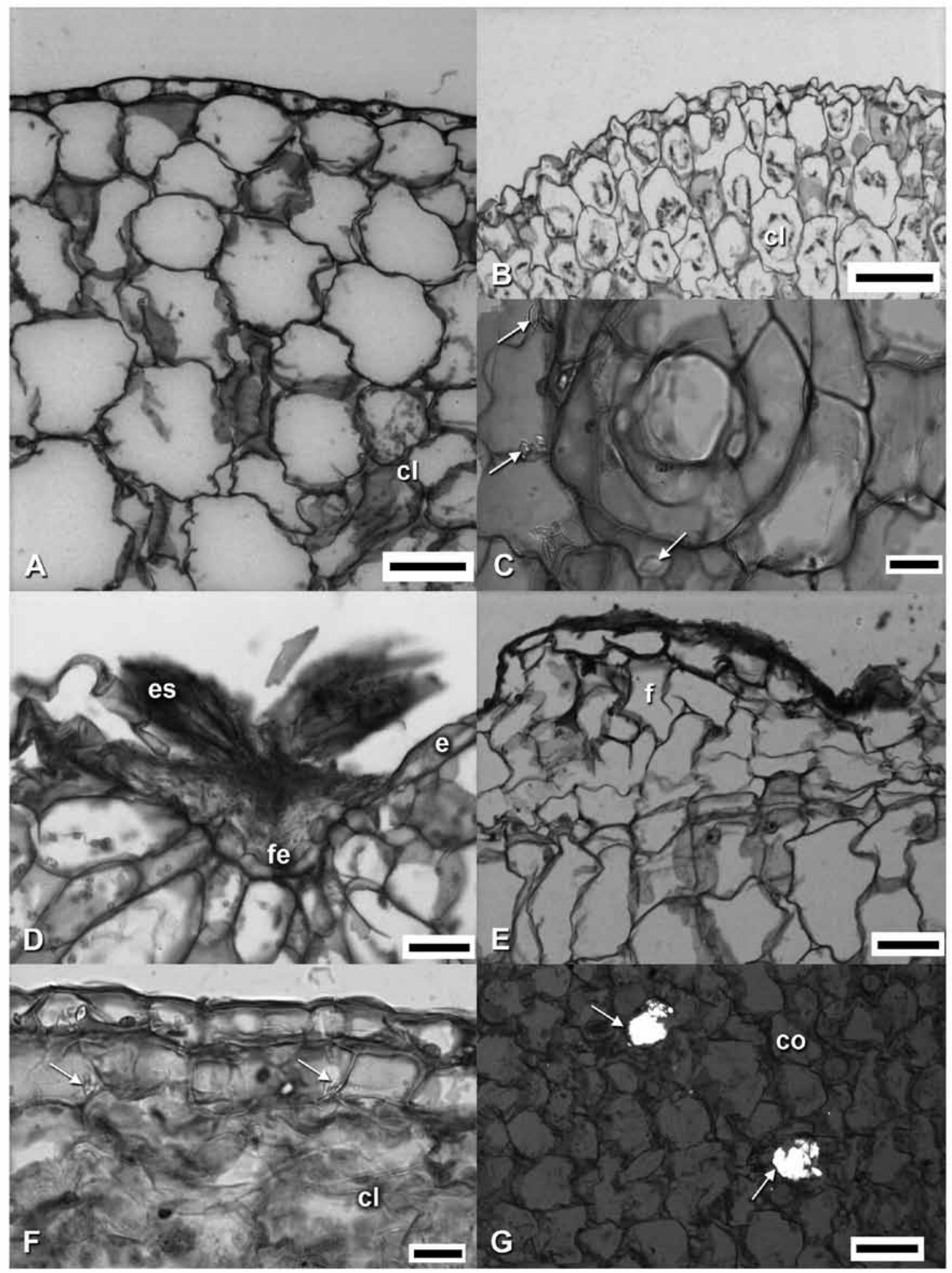

Figura 5. Epicótilo y tallo en plántulas y plantas jóvenes de Pachycereeae, cortes transversales (T). A. Epidermis simple en Pachycereus grandis, dos meses de edad. B. Epidermis papilosa, Neobuxbaumia mezcalaensis, dos meses de edad. C. Cristales prismáticos en células epidérmicas, vista paradermal, en Neobuxbaumia multiareolata, 12 meses de edad. D. Areóla con espinas en Neobuxbaumia multiareolata, 14 meses de edad. E. Formación de peridermis Myrtillocactus geometrizans, 12 meses de edad. F. Hipodermis con cristales en Neobuxbaumia multiareolata, 24 meses de edad. G. Drusas en córtex con luz polarizada en Pachycereus grandis, seis meses de edad. Barra $100 \mu \mathrm{m}$ en A, B; de $50 \mu \mathrm{m}$ en D, E, de $20 \mu \mathrm{m}$ en C, F; de $100 \mu \mathrm{m}$ en G. cl, clorénquima; co, córtex; e, epidermis; es, espina; f, felema; fe, felógeno; flechas, cristales prismáticos y drusas. 


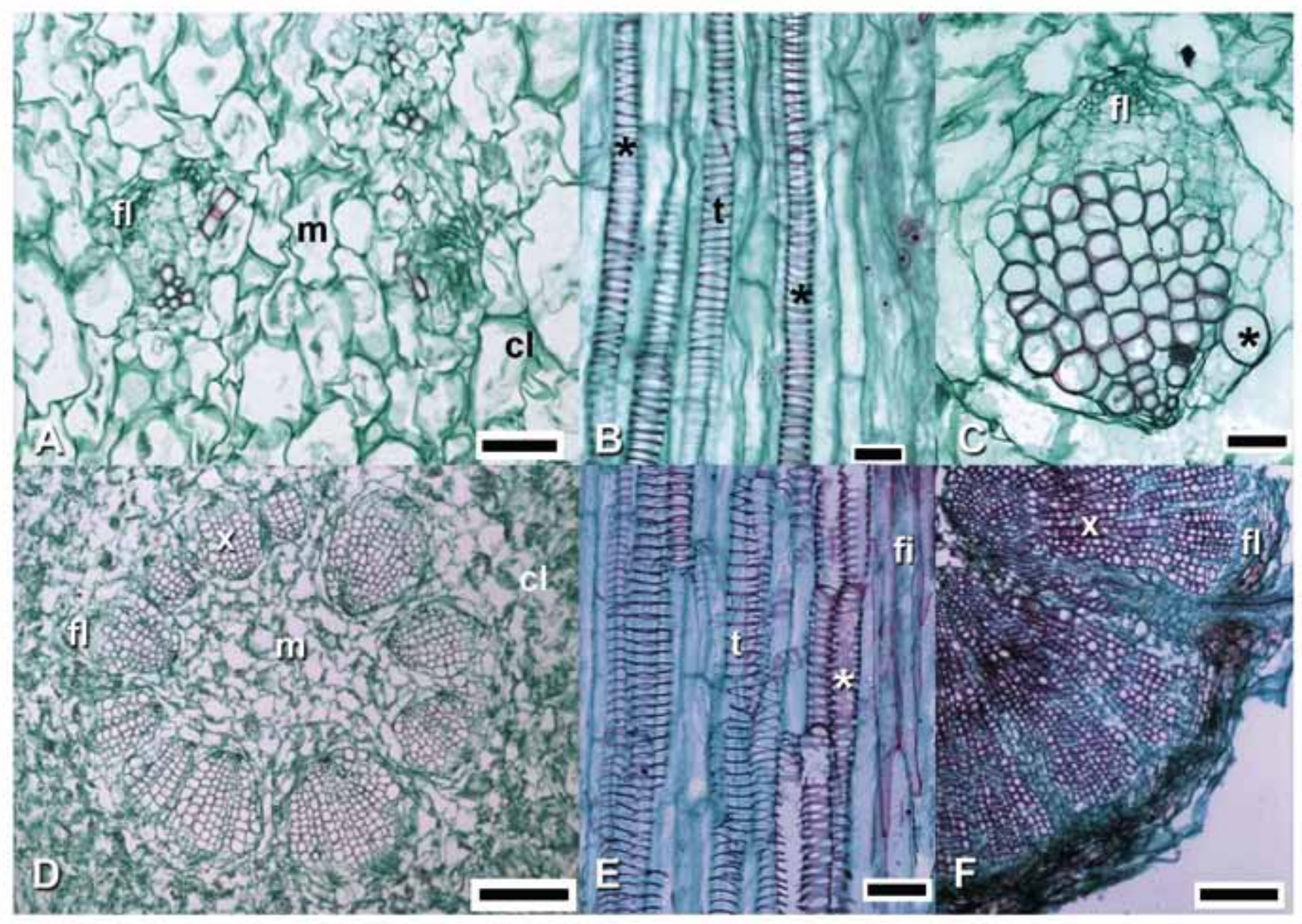

Figura 6. Tejido vascular de epicótilo y tallo en plántulas y plantas jóvenes de Pachycereeae, cortes transversales (T) y longitudinales (L). A. Estela (T) en Myrtillocactus geometrizans, seis meses de edad. B. Elementos de vaso y traqueidas de banda ancha (L) en Neobuxbaumia multiareolata, dos meses de edad. C. Cámbium vascular (T) en Pachycereus grandis, nueve meses de edad. D. Cilindro vascular con crecimiento secundario (T) en Neobuxbaumia mezcalaensis, 12 meses de edad. E. Elementos de vaso con paredes reticuladas y punteaduras pseudoescalariformes y fibras en Pachycereus grandis, 24 meses de edad. F. Madera fibrosa en Pachycereus grandis, 24 meses de edad. Barra de $50 \mu \mathrm{m}$ en A, C, E; de $20 \mu \mathrm{m}$ en B, de $300 \mu \mathrm{m}$ en D, F. cl, clorénquima; fi, fibra; fl, floema, m, médula, t, traqueida de banda ancha, $\mathrm{x}$, xilema secundario; *, elementos de vaso.

felógeno cambia desde la raíz hacia la porción inferior de la zona de transición y en la zona de transición. Mientras en la raíz el felógeno tiene origen en el periciclo, en el hipocótilo se origina de células del parénquima subyacentes a la epidermis.

Cotiledones.- Los cotiledones presentan epidermis simple (Figura 4D) y paredes anticlinales onduladas vistas en superficie, y estomas escasos. La mayor parte de los cotiledones es mesofilo, con abundantes cloroplastos, espacios intercelulares y granos de almidón sólo en Pachycereus grandis. El procámbium, presente en los cotiledones desde la etapa de embrión, forma tejido vascular escaso constituido por xilema y floema. Después de 12 meses de crecimiento, las plántulas conservan los cotiledones con peridermis y colapso de la mayoría de las células de parénquima (Figura 4E). Epicótilo-tallo.- La epidermis es simple con la cutícula delgada $(<1 \mu \mathrm{m})$, sus células son comúnmente tabicadas o papilosas en Neobuxbaumia (Figura 5A, B), pero vistas en superficie tienen paredes anticlinales onduladas. La epidermis en plántulas de 12 meses o más de edad de especies de Neobuxbaumia, presenta cristales prismáticos pequeños (Figura 5C). Los estomas son paracíticos, asociados a cámaras subestomáticas poco profundas. En las aréolas, las espinas son producidas por un meristemo basal de espina y sus células llegan a esclerificarse; las espinas se fusionan en su base por la formación de felógeno entre ellas (Figura 5D). Raramente puede observarse la formación de peridermis, la cual comienza como parches pequeños en algunas partes del tallo en plántulas de nueve o más meses de edad (Figura $5 \mathrm{E})$. La hipodermis tiene uno o dos estratos, es no colenquimatosa hasta los 24 meses de edad y sin ningún tipo de contenido, excepto en especies de Neobuxbaumia que pre- 
sentan pequeños cristales prismáticos (Figura 5F). El tejido cortical está formado por clorénquima laxo, con espacios intercelulares, excepto en $P$. grandis que a los nueve meses presenta las células típicas en empalizada. Células de mucílago pequeñas son visibles en el tejido cortical de plántulas desde el primer mes de edad y las drusas son evidentes hasta los seis meses de edad únicamente en Pachycereus grandis (Figura 5G). Los haces vasculares corticales son colaterales, sin crecimiento secundario en plántulas de 12 meses o más de edad.

La estela tiene de cuatro a cinco haces vasculares colaterales en Myrtillocactus geometrizans (Figura 6A) hasta 17 en Pachycereus grandis. Cada haz está compuesto de xilema primario con traqueidas de banda ancha y vasos escasos; cada elemento de vaso tiene paredes secundarias con engrosamientos anulares y helicoidales (Figura 6B); el floema primario presenta elementos de tubo criboso con placas cribosas simples y células acompañantes. El cámbium vascular es evidente y se restringe a los haces vasculares en plántulas de 12 meses de edad de la mayoría de las especies y de nueve meses en $P$. grandis (Figura 6C) y Stenocereus queretaroensis. Conforme se incrementa la edad de las plántulas se observan divisiones en las células que constituyen el cámbium interfascicular. Particularmente en especies de Neobuxbaumia, la acumulación de xilema secundario es relativamente escasa, los haces permanecen discretos y no llegan a fusionarse para formar un cilindro vascular sólido (Figura 6D). En contraste, en plántulas de $P$. grandis y $S$. queretaroensis con crecimiento más vigoroso, el cámbium vascular forma un cilindro sólido en plantas de 24 meses de edad (Figura 6F). El xilema secundario de todas las especies está formado por elementos de vaso con pared secundaria reticulada o punteaduras pseudoescalariformes, células de parénquima no lignificado y fibras sólo en algunas especies como $P$. grandis (Figura 6E). También a los 24 meses, la parte basal del tallo de la mayoría de las especies forma peridermis con varios estratos de células lignificadas y células del parénquima cortical totalmente colapsadas. La médula está constituida por células de parénquima sin inclusiones celulares y con pequeños y escasos haces medulares en Neobuxbaumia y $P$. grandis.

\section{Discusión}

Morfología de plántulas. Las plántulas de las especies estudiadas presentaron una morfología similar a la registrada en otras Cactaceae (Buxbaum, 1955; Meyrán, 1956; Gibson, 1988a, b, 1990a, b); algunas diferencias morfológicas durante el crecimiento temprano permitieron distinguir las especies. Por ejemplo, en Pachycereus grandis los cotiledones son desiguales, largos y suculentos, en comparación con las otras especies estudiadas donde son cónicos y pequeños, además los cotiledones tienen ápices redondeados exclusivamente en las especies de Neobuxbaumia y puntiagudos en
Myrtillocactus geometrizans. Con respecto al hipocótilo, es cilíndrico y corto en la mayoría de las especies; sin embargo, Neobuxbaumia multiareolata se distingue por tener un hipocótilo relativamente delgado y largo. Las plántulas de N. mezcalaensis y Stenocereus queretaroensis se caracterizaron por presentar una coloración rojiza durante las primeras semanas de su crecimiento.

La reducción en tamaño de los cotiledones en las especies estudiadas coincide con lo que se denominó "metamorfosis ontogenética": los cotiledones se ensanchan hacia la base y se fusionan gradualmente con el tallo hasta ser totalmente indistinguibles (Ganong, 1898). Los cotiledones se reducen a diferentes edades y no se relacionan con el tamaño de la semilla, por ejemplo en Escontria chiotilla son imperceptibles a los 24 meses, mientras que en Myrtillocactus geometrizans a los 12 y en Pachycereus grandis a los 14 meses. Esta característica morfológica podría ser uno de los marcadores morfológicos para establecer cuando finaliza el estadio de plántula e inicia la fase del ciclo de vida como individuo juvenil.

El cambio de un epicótilo globoso a cilíndrico en las especies de Pachycereeae no sólo se evidenció por la proporción alto $v s$ diámetro sino también por la presencia de los tubérculos y costillas. Al igual que como señaló Boke (1952, 1957), los primordios de tubérculos se desarrollaron de manera acrópeta, a ambos lados del meristemo apical de la plántula. Los podarios que alternan con los cotiledones al principio, van formando pequeños tubérculos con sus aréolas axilares y finalmente, por disposición de tubérculos en hileras verticales se desarrollan las costillas. Esta disposición determinada de los tubérculos se denomina dispersa y fue observada en las especies estudiadas. De acuerdo a Gibson y Nobel (1986), la transición de una forma globosa a una forma cilíndrica del tallo puede tomar varios años. Esto no ocurrió en las especies estudiadas, el ordenamiento de tubérculos en hileras verticales para constituir las costillas típicas de especies columnares se observó generalmente a los 12 meses de edad (Cuadro 3). La presencia de las costillas y la desaparición de los cotiledones, ayudarían a reconocer la fase de juvenil en las especies de columnares de la tribu Pachycereeae.

El crecimiento y desarrollo de las plántulas fue relativamente lento como ocurre en Cactaceae (Gibson y Nobel, 1986). Un retraso notable en el desarrollo del epicótilo fue característico de especies provenientes de semillas pequeñas (<0.70 mg, Loza-Cornejo et al., 2008) como Escontria chiotilla y Myrtillocactus geometrizans. La altura de las plántulas de un año de edad de las especies estudiadas tuvo una media que varió de poco más de $1 \mathrm{~cm}$ en $E$. chiotilla y M. geometrizans hasta 10-12 cm en Neobuxbaumia multiareolata y Pachycereus grandis. Otras especies de la misma tribu también muestran crecimiento lento en el primer o segundo año de vida; por ejemplo, para Carnegiea gigantea se menciona una altura menor a $2.5 \mathrm{~cm}$ (Despain, 1974; Jordan 
Cuadro 3. Eventos morfo-anatómicos de las especies de Pachycereeae estudiadas. c.v., cámbium vascular; e., epicótilo.

\begin{tabular}{|c|c|c|c|c|c|c|c|}
\hline \multicolumn{8}{|c|}{ Meses } \\
\hline Especie & $1-2$ & 4 & 6 & 9 & 12 & 14 & 24 \\
\hline Escontria & $\begin{array}{l}\text { e. visible a los } \\
\text { dos meses }\end{array}$ & & $\begin{array}{l}\text { inicio formación } \\
\text { de podarios }\end{array}$ & & $\begin{array}{l}\text { e. globoso con } \\
\text { podarios desorde- } \\
\text { nados, cotiledones } \\
\text { turgentes, estableci- } \\
\text { miento de c.v. }\end{array}$ & & $\begin{array}{l}\text { reducción de } \\
\text { cotiledones, e. } \\
\text { con } 5 \text { costillas, }\end{array}$ \\
\hline Myrtillocactus & - & $\begin{array}{l}\text { e. visible, haciendo } \\
\text { más delgados los } \\
\text { cotiledones y con } \\
\text { ápices puntiagudos }\end{array}$ & $\begin{array}{l}\text { e. más o menos } \\
\text { cilíndrico, tubérculos } \\
\text { desordenados y } \\
\text { dispersos }\end{array}$ & & $\begin{array}{l}\text { reducción de los } \\
\text { cotiledones, estable- } \\
\text { cimiento c.v. }\end{array}$ & $\begin{array}{l}\text { e. inicio de } \\
\text { diferenciación } \\
\text { de costillas }\end{array}$ & $\begin{array}{l}\text { e. cilíndrico } \\
\text { con } 5 \text { costillas }\end{array}$ \\
\hline Neobuxbaumia & $\begin{array}{l}\text { e. globoso al } \\
\text { mes }\end{array}$ & & $\begin{array}{l}\text { e. cilíndrico con } \\
\text { tubérculos desor- } \\
\text { denados }\end{array}$ & & $\begin{array}{l}\text { e. con } 10-13 \\
\text { costillas, estable- } \\
\text { cimiento de c. v. }\end{array}$ & $\begin{array}{l}\text { reducción de } \\
\text { los cotiledones }\end{array}$ & $\begin{array}{l}\text { presencia de } \\
\text { haces medulares } \\
\text { y cristales en } \\
\text { epidermis e } \\
\text { hipodermis }\end{array}$ \\
\hline Pachycereus & $\begin{array}{l}\text { e. visible al } \\
\text { mes }\end{array}$ & $\begin{array}{l}\text { e. cilíndrico con } \\
\text { tubérculos desor- } \\
\text { denados }\end{array}$ & & $\begin{array}{l}\text { establecimiento } \\
\text { c.v. }\end{array}$ & e. con 8 costillas & $\begin{array}{l}\text { reducción de } \\
\text { los cotiledones }\end{array}$ & $\begin{array}{l}\text { presencia de } \\
\text { haces medulares }\end{array}$ \\
\hline Stenocereus & $\begin{array}{l}\text { e. visible al } \\
\text { mes }\end{array}$ & $\begin{array}{l}\text { e. con tubérculos } \\
\text { desordenados }\end{array}$ & $\begin{array}{l}\text { e. cilíndrico con } \\
\text { tubérculos } \\
\text { desordenados }\end{array}$ & $\begin{array}{l}\text { establecimiento } \\
\text { c.v. }\end{array}$ & $\begin{array}{l}\text { reducción de los } \\
\text { cotiledones, no } \\
\text { distinguibles }\end{array}$ & $\begin{array}{l}\text { tallo con siete } \\
\text { costillas }\end{array}$ & \\
\hline
\end{tabular}

y Nobel, 1981) y para P. pringlei, Stenocereus gummosus y $S$. thurberi se registra una altura de 20 a $30 \mathrm{~cm}$ a $l o s$ dos años de edad (Nerd et al., 1993).

Una vez germinadas las semillas, la raíz primaria no tuvo un desarrollo extenso, que podría estar asociado a su crecimiento determinado (Dubrovsky, 1997), pero si se observaron abundantes pelos microscópicos, que tienen un papel importante en la absorción de agua y nutrimentos en diversas especies (Charlton, 1991). En etapas posteriores del desarrollo, hay un mayor crecimiento de raíces laterales que pueden incrementar el área de contacto entre el sistema radical y las partículas del suelo, lo cual probablemente es un requisito esencial para el establecimiento de la plántula en el campo. Ambas raíces (primaria y secundarias) forman tempranamente peridermis. Nobel (1994) señala que la acumulación de suberina en la peridermis se compensa generalmente con un incremento en el número de vasos de xilema a medida que aumenta el crecimiento secundario de este órgano, lo cual se observó en las especies estudiadas.

De acuerdo a Hunt y Nobel (1987) el crecimiento del tallo y raíz se presenta en proporción similar en plántulas de especies suculentas como Agave deserti y Ferocactus acanthodes. Éste pudiera ser el caso de dos de las especies estudiadas en el presente trabajo, Pachycereus grandis y Stenocereus queretaroensis, ya que se caracterizaron por tener las plántulas más vigorosas (mayor crecimiento de la raíz y tallo). Estos autores afirman que una mayor longitud de raíz y una mayor altura del tallo con menor área superficial puede ser un factor clave para el establecimiento de plántulas en el campo. En el caso de la raíz, ésta ayudaría para el anclaje con firmeza de la plántula al sustrato; mientras que para el tallo, la proporción más baja de superficie a volumen probablemente reduciría la capacidad de absorción de dióxido de carbono, pero no la absorción de la luz y ello realmente sería beneficioso para la conservación del agua y establecerse antes de iniciar la época desfavorable en los bosques secos o matorrales donde habitan; sin embargo, se requieren estudios adicionales en campo para concluir al respecto.

Anatomía de plántulas. Eje hipocótilo-raíz-cotiledones.- La disposición del tejido vascular en el hipocótilo de las especies estudiadas fue similar a Opuntia (Freeman, 1969; Hamilton, 1970a); con dos haces vasculares colaterales discretos, que conforme incrementa la edad de la plántula, presentan mayor acumulación de elementos traqueales en el xilema. En plántulas de Pachycereus grandis de un año de edad, hay notoria acumulación de xilema secundario fibroso en la parte basal del hipocótilo. La presencia de fibras no se asoció con el tamaño de la plántula debido a que las plántulas de Neobuxbaumia que también son altas como las de $P$. grandis, no desarrollaron fibras a esa edad. Las fibras libriformes en $P$. grandis probablemente desempeñan una función importante para el soporte del cuerpo de la plántula, lo que facilitaría su establecimiento en campo. Los cotiledones se caracterizaron por la ausencia de inclusiones minerales, escasez de estomas y formación temprana de peridermis y persistieron como una estructura colapsada con una peridermis que tendría una función importante en regular la econo- 
mía hídrica en el ambiente estacional donde se desarrollan, al aislar esta región de la plántula con las células de súber. Esto es, la peridermis constituye una barrera impermeable que junto con otros atributos morfo-fisiológicos permitiría a la plántula sobrevivir en limitaciones medio ambientales, particularmente la escasez de agua e influir así, en patrones de abundancia y distribución como se presenta en plántulas de otras especies (Molinas y Verdaguer, 1993).

Tallo.- La estructura anatómica de la epidermis contrasta con la anatomía de este tejido en tallos adultos. En éstos la cutícula tiene un mayor espesor, las células epidérmicas tienen paredes anticlinales rectas y divisiones secundarias (Loza-Cornejo y Terrazas, 2003); mientras que en las plántulas estudiadas, la epidermis tiene células de pared anticlinal ondulada, estomas escasos y cutícula delgada. Estas características del tejido dérmico seguramente le dan la capacidad de ganar y perder turgencia fácilmente como se ha señalado para Eryosyce (Nyffeler y Eggli, 1997). La mayoría de las inclusiones minerales registradas para Pachycereeae (Terrazas y Loza-Cornejo, 2002) no se presentan en las plántulas, excepto en Neobuxbaumia, donde si se observaron cristales prismáticos pequeños en células de la epidermis e hipodermis del tallo, como se describieron en plantas adultas.

La presencia de espinas que se originan del meristemo de aréolas fue una característica típica de las plántulas. Además, las células maduras de la zona basal meristemática de la espina forman paredes gruesas, y luego las bases de las espinas individuales se fusionan con la formación de corcho entre ellas. Esta agrupación constituye una defensa efectiva para evitar la separación de espinas individuales; además, el desarrollo de una cubierta de corcho cubriendo la superficie de aréolas disminuiría la pérdida de agua (Gibson y Nobel, 1986). Una diferencia también importante entre plantas adultas y plántulas fue la ausencia de hipodermis colenquimatosa en estas últimas. Las células de una hipodermis colenquimatosa contienen altas concentraciones de pectina y hemicelulosa e intervienen en funciones de soporte, además de representar una barrera contra patógenos en la planta adulta (Terrazas y Mauseth, 2002; Mauseth, 2006), mientras que en una planta joven, debido a su lento crecimiento, la diferenciación de una hipodermis con dichas características es un proceso también lento y transcurren varios años para lograrla. El desarrollo de traqueidas de banda ancha y elementos de vaso con paredes secundarias con engrosamientos anulares y helicoidales en el xilema secundario de las plántulas se interpreta como una adaptación a la habilidad de la plántula para deshidratarse sin dañar el tejido vascular. Una vez establecida la plántula, los requerimientos para dar rigidez al tallo en crecimiento se incrementan, modificándose la "señal ontogenética" para diferenciar las fibras y los elementos de vasos con paredes secundarias reticuladas o punteadas; tipos celulares típicos de las plantas adultas. Este cambio se ha mencionado para otras Cactoideae (LozaCornejo et al., 2003; Mauseth, 2004; Romero-Godofredo y
Melo-de Pinna, 2008). Una disposición característica de la estela con haces vasculares que permanecen sin fusionarse, se observó sólo en las plántulas de Neobuxbaumia. Esta disposición es típica de los individuos adultos de las especies de este género (Terrazas y Loza-Cornejo, 2002) y de acuerdo a varios autores permite un alargamiento de la médula y a su vez, un incremento en anchura de los radios primarios (Gibson y Nobel, 1986). Una característica distintiva de la médula en Cactoideae es la presencia de haces medulares. Estos únicamente se observaron en plántulas de Neobuxbaumia y Pachycereus grandis; seguramente en las otras especies su desarrollo es tardío, ya que si se han registrado en individuos adultos. Gibson y Nobel (1986) mencionan que el sistema de haces medulares tiene una función importante en el suministro de agua y compuestos elaborados a las células y probablemente interviene en la función de almacén de almidón en este tejido. Consideramos que su presencia en los individuos de 24 meses es una evidencia adicional al cambio de fase en el ciclo de vida de estas especies.

Concluimos que las plántulas más vigorosas, con mayor crecimiento de la raíz, mayor tamaño de cotiledones y de tallo, distinguen a Pachycereus grandis. En el caso de plántulas de Escontria chiotilla y Myrtillocactus geometrizans, el menor incremento en tamaño se debió probablemente a la menor asignación de biomasa a tallo y raíz, ya que son provenientes de semillas pequeñas. Ésta podría ser una estrategia adaptativa de supervivencia que se refleja en una menor tasa de transpiración a través de tallos pequeños con menor número de costillas. Además, el retraso en el crecimiento de estas especies coincide con la presencia de metabolismo $\mathrm{C}_{3}$ durante las primeras semanas de edad de las plántulas (Loza-Cornejo, 2004), y probablemente las ayudarían a tolerar sequías de corto plazo que se llegan a presentar en los bosques secos durante la estación lluviosa.

La extrapolación del conjunto de caracteres interespecíficos de plántulas crecidas en laboratorio a plantas adultas en el campo o viceversa debe ser establecida con prudencia. Investigación adicional seguramente confirmaría la correlación entre el desempeño morfo-funcional de la plántula y el tamaño de la semilla, la función fotosintética y/o almacén de reserva de cotiledones aquí sugeridos. Los caracteres morfológicos como ausencia de cotiledones y diferenciación de costillas en el tallo, junto con la diferenciación de cámbium vascular, de más elementos lignificados (fibras) en xilema del tallo y raíz y de haces medulares, se consideran aspectos importantes para establecer el límite entre plántula y planta joven en las especies de Pachycereeae estudiadas.

\section{Literatura citada}

Anderson E.F. 2001. The Cactus Family. Timber Press, Portland. Berlyn G.P. y Miksche J.P. 1976. Botanical Microtechnique and Cytochemistry. The Iowa State University Press, Ames.

Boke N.H. 1952. Leaf and areole development in Coryphantha. American Journal of Botany 39:134-145. 
Boke N.H. 1957. Structure and development of the shoot in Toumeya. American Journal of Botany 44:888-896.

Boke N.H. 1959. Endomorphic and ectomorphic characters in $\mathrm{Pe}$ lecyphora and Encephalocarpus. American Journal of Botany 46:197-209.

Burger H.D. 1972. Seedling of Some Tropical Trees and Shrubs Mainly of South East Asia. Centre for Agricultural Publishing and Documentation, Wageningen.

Buxbaum F. 1955. Morphology of Cacti. Section III. Fruits and Seeds. Abbey Garden Press, Pasadena.

Buxbaum F. 1961. Die entwicklungslinien der tribes Pachycereeae F. Buxb. (Cactaceae-Cereoideae). Botanische studien 12:1-107.

Charlton W.A. 1991. Lateral root initiation. En: Waisel Y. Eshel A y Kafkafi U. Eds. Plant-Roots- The Hidden Half, pp. 103-128. Marcel Dekker, Inc., Nueva York.

Despain D.G. 1974. The survival of saguaro (Carnegiea gigantea) seedlings on soils of differing albedo and cover. Journal of Arizona Academy of Sciences 9:102-107.

De Vogel E.F. 1980. Seedlings of Dicotyledons Structure, Development, Types: Description of 150 Woody Malesian Taxa. Centre for Agricultural Publishing and Documentation, Wageningen.

Dubrovsky J.G. 1996. Seed hydration memory in Sonoran Desert cacti and its ecological implication. American Journal of Botany 83:624-632.

Dubrovsky J.G. 1997. Determinate primary-root growth in seedlings of Sonoran Desert Cactaceae, its organization, cellular basis, and ecological significance. Planta 203:85-92.

Dubrovsky J.G., North G.B. y Nobel P.S. 1998. Root growth, developmental changes in the apex, and hydraulic conductivity for Opuntia ficus-indica during drought. New Phytologist 138:7582.

Freeman T.P. 1969. The developmental anatomy of Opuntia basilaris I. Embryo, root and transition zone. American Journal of Botany 56:1067-1074.

Freeman T.P. 1973. Developmental anatomy of epidermal and mesophyll chloroplasts in Opuntia basilaris leaves. American Journal of Botany 60:86-91.

Ganong W.F. 1898. Contributions to knowledge of the morphology and ecology of the Cactaceae. II. The comparative morphology of the embryos and seedlings. Annals of Botany 12:423-474.

Gibson A.C. 1988a. The systematics and evolution of subtribe Stenocereinae 3. Myrtillocactus. Cactus and Succulent Journal (U.S.) 60:109-116.

Gibson A.C. 1988b. The systematics and evolution of subtribe Stenocereinae 4. Escontria. Cactus and Succulent Journal (U.S.) 60:161-167.

Gibson A.C. 1989a. The systematics and evolution of subtribe Stenocereinae. 6. Stenocereus stellatus and Stenocereus treleasei. Cactus and Succulent Journal (U.S.) 61:26-32.

Gibson A.C. 1989b. The systematics and evolution of subtribe Stenocereinae.7. The Machaerocerei of Stenocereus. Cactus and Succulent Journal (U.S.) 61:104-112.

Gibson A.C. 1990a. The systematics and evolution of subtribe Stenocereinae. 8. Organ pipe cactus and its closest relatives. Cactus and Succulent Journal (U.S.) 62:13-24.

Gibson A.C. 1990b. The systematics and evolution of subtribe Stenocereinae. 9. Stenocereus queretaroensis and its closest relatives. Cactus and Succulent Journal (U.S.) 62:170-176.

Gibson A.C. y Nobel P.S. 1986. The Cactus Primer. Harvard University Press, Cambridge.
Gutiérrez de la Rosa A., Reyes S.J. y Brachet C. 1999. Morfología comparada de plántulas de 4 especies del género Mammillaria Serie Longiflorae Hunt. En Vázquez Dávila, M.A. Ed. Resúmenes II Congreso Mexicano y I Congreso Latinoamericano y del Caribe sobre Cactáceas y otras Plantas Suculentas, pp. 55, Sociedad Mexicana de Cactología, Oaxaca.

Hamilton M.W. 1970a. Seedling development of Opuntia bradtiana (Cactaceae). American Journal of Botany 57:599-603.

Hamilton M.W. 1970b. The comparative morphology of three cylindropuntias. American Journal of Botany 57:1255-1263.

Hunt E.R. y Nobel P.S. 1987. Allometric root/shoot relationships and predicted water uptake for desert succulents. Annals of Botany 59:571-577.

Jordan P.W. y Nobel P.S. 1981. Seedling establishment of Ferocactus acanthodes in relation to drought. Ecology 62:901-906.

Leroy C., Jauneau A., Quilichini A., Dejean A. y Orivel J. 2010. Comparative structure and ontogeny of the foliar domatia in three Neotropical myrmecophytes. American Journal of Botany 97:557-565.

Loza-Cornejo S. 2004. Características de semillas, germinación y desarrollo de plántulas de seis especies de Pachycereeae (Cactaceae). Tesis de Doctorado en Ciencias. Programa de Botánica, Colegio de Postgraduados. Montecillo, Estado de México. $157 \mathrm{p}$.

Loza-Cornejo S. y Terrazas T. 2003. Epidermal and hypodermal characteristics in NorthAmerican Cactoideae (Cactaceae). Journal of Plant Research 116:27-35.

Loza-Cornejo S., Terrazas T., López-Mata L. y Trejo C. 2003. Características morfo-anatómicas y metabolismo fotosintético de plántulas de Stenocereus queretaroensis (Cactaceae): su significado adaptativo. Interciencia 28:83-89.

Loza-Cornejo S., López-Mata L. y Terrazas T. 2008. Morphological seed traits and germination of six species of Pachycereeae (Cactaceae). Journal of the Professional Association for Cactus Development 10:71-84.

Mauseth J.D. 1978. An investigation of the morphogenetic mechanisms which control the development of zonation in seedlings shoot apical meristems. American Journal of Botany 65:158167.

Mauseth J.D. 1979. Cytokining-elicited formation of the pith-rib meristem and other effects of growth regulators on the morphogenesis of Echinocereus (Cactaceae) seedlings shoot apical meristems. American Journal of Botany 66:446-451.

Mauseth J.D. 1980. A morphometric study of the ultrastructure of Echinocereus engelmanii (Cactaceae). I. Shoot apical meristems at germination. American Journal of Botany 67:173-181.

Mauseth J.D. 2004. Wide-band tracheids are present in almost all species of Cactaceae. Journal of Plant Research 117:69-76.

Mauseth J.D. 2006. Structure-function relationships in highly modified shoots of Cactaceae. Annals of Botany 98: 901-926.

Meyrán J. 1956. Notas sobre plántulas de cactáceas. Cactáceas y Suculentas Mexicanas 1:107-112.

Molinas M.L. y Verdaguer D. 1993. Lignotuber ontogeny in the cork-oak (Quercus suber; Fagaceae) II. Germination and young seedling. American Journal of Botany 80:182-191.

Nerd A., Raveh E. y Mizrahi Y. 1993. Adaptation of five columnar cactus species to various conditions in the Negev Desert of Israel. Economic Botany 47:304-311.

Nobel P.S. 1994. Remarkable Agaves and Cacti. Oxford University Press, Nueva York. 
Nyffeler R. y Eggli U. 1997. Comparative stem anatomy and systematics of Eriosyce sensu lato (Cactaceae). Annals of Botany 80:767-786.

Pérez-García B. y Mendoza A. 2002. Morfología vegetal neotropical. Revista Biología Tropical 50:893-902.

Ricardi M., Hernández C. y Torres F. M. 1987. Morfología de Plántulas de Árboles de los Bosques del Estado Mérida. Talleres Gráficos de la Universidad de Los Andes, Mérida.

Romero-Godofredo V. y Melo-de Pinna G.F. 2008. Occurrence of wide-band tracheids in Cactaceae: wood variation during Pilosocereus aurisetus development. Journal of the Torrey Botanical Society 135:94-102.

San Miguel-Chávez R., Soto-Hernández M., Terrazas T. y Kite G. 2006. Morphology and alkaloidal profile between seedlings of Erythrina americana Miller and E. coralloides A. DC. Feddes Repertorium 117:232-239.

SAS Institute. 2008. Statistical Analysis System. Release 9.2 SAS Institute Inc., Cary.

Terrazas T. y Loza-Cornejo S. 2002. Phylogenetic relationships of
Pachycereeae: a cladistic analysis based on anatomical-morphological data. En: Fleming T.H. y Valiente-Banuet A. Eds. Evolution, Ecology, and Conservation of the Columnar Cacti and their Mutualist, pp. 66-86, The University Arizona Press, Tucson.

Terrazas T. y Mauseth J.D. 2002. Shoot anatomy and morphology. En: Nobel P. S. Ed. The Cacti: Biology and Uses, pp. 23-40, University of California Press, Berkeley.

Vega-Villasante F., Nolasco H., Montaño C., Romero-Schmidt H.L. y Vega-Villasante E. 1996. Efecto de la temperatura, acidez, iluminación, salinidad, irradiación solar y humedad sobre la germinación de semillas de Pachycereus pecten-aboriginum "cardón barbón" (Cactaceae). Cactáceas y Suculentas Mexicanas 41:51-61.

Zevallos-Pollito P.A. y Flores-Bendezú Y. 2003. Caracterización morfólogica de plántulas de "uña de gato" Uncaria tomentosa (Willdernow ex Roemer \& Schultes) D.C. y U. guianensis (Aublet) Gmelin del Bosque Nacional Alexander von Humboldt. Ecología Aplicada 2:41- 46.

Recibido: 20 de diciembre de 2010

Aceptado: 12 de abril de 2011

Editora: Dra. Victoria Sosa 